\title{
Robot-assisted surgical treatment in a case of superior mesenteric artery syndrome
}

\author{
Jordan Ho, Herman Bami
}

\begin{abstract}
We present a case of superior mesenteric artery syndrome in a 14-year-old female. The patient was initially managed using dietary changes and postprandial assumption of the left lateral decubitus position. However, after 2 months with no improvement, the patient was treated using a robot-assisted Strong's procedure. The patient fully recovered within 1 year. We further discuss superior mesenteric artery syndrome and its current treatment practices, and give a general overview of the current state of robot-assisted surgery.
\end{abstract}

\section{CASE PRESENTATION}

A 14-year-old female was referred to the general surgery department with early satiety, postprandial vomiting and epigastric pain, which improved in the left lateral decubitus position. She reported a $10 \mathrm{~kg}$ weight loss in the previous 3 months. There was no history of nutritional disorders, upper gastrointestinal symptoms, or any other medical conditions. Other than the stated symptoms and her low body mass index (BMI), the patient was relatively healthy. No use of pain medication was mentioned.

An upper gastrointestinal endoscopy was performed, showing neither pyloric stricture nor inflammation of the stomach; however, there was external compression of the third portion of the duodenum. A subsequent computed tomography (CT) scan showed a dilated stomach and proximal duodenum, and computed imaging reconstruction showed a narrowed aortomesenteric angle with a pathognomonic short distance of $3 \mathrm{~mm}$ between the aorta and the superior mesenteric artery. This confirmed the diagnosis of superior mesenteric artery syndrome.

The patient was unwilling to be hospitalized and refused parenteral nutrition; thus she was initially sent home with instructions to take small, liquefied, high-caloric meals and to stay in the left lateral decubitus position after meals. After 2 months, the patient reported slight pain relief, but as the treatment yielded neither weight gain nor radiologic improvement, surgical treatment was decided.

\section{SURGERY}

To avoid complications of anastomotic bypass procedures, and to maintain the integrity of the gastrointestinal tract, it was decided that the preferred surgical treatment for the patient was a Strong's procedure. Robot-assisted surgery using the da Vinci system was used to provide a minimally invasive approach.

For this laparoscopic approach, four ports were used. A camera port was placed inferior to the umbilicus; two robotic trocars were placed bilaterally at the level of the umbilicus at the mid-clavicular line; and one subcostal trocar was placed at the left anterior axillary line. The surgeon identified the dilated third portion of the duodenum and mobilized it by dissecting its retroperitoneal attachments and dividing the ligament of Treitz. The fourth portion of the duodenum and the duodenojejunal junction were then peritonised to allow for their mobilization. The junction was brought to the right of the superior mesenteric vessels and dissected from its attachments. Finally, a full circumferential dissection of the superior mesenteric axis and the aortomesenteric space allowed the proximal jejunal loops to be translocated to the right of the superior mesenteric artery and sutured in place. The entire operation lasted 1 hour and 15 minutes, with insignificant intraoperative blood loss.

\section{CASE OUTCOME}

The patient was fully mobilized a few hours after the surgery, although the nasogastric tube was kept for 24 hours. An upper gastrointestinal series confirmed free passage of contrast to the jejunum, and oral fluid intake was initiated the next day. The patient was discharged from the hospital uneventfully by the third postoperative day. By three months, the patient had full clinical and radiological regression of her manifestations and significant weight gain. By 1 year, the patient had fully recovered and was symptomfree.

\section{DISCUSSION}

\section{Superior Mesenteric Artery Syndrome}

Superior mesenteric artery (SMA) syndrome, also called Wilkie's syndrome, was first described in 1842 by Carl von Rokitansky. Sir David Wilkie published the first case series of SMA syndrome in 1927, describing 75 patients. ${ }^{1}$ SMA syndrome is caused by compression of the duodenum between the aorta and the superior mesenteric artery, where the resulting inadequate aortomesenteric angle causes obstruction of the duodenum. A normal aortomesenteric angle is between 380 and 560 degrees, and the aortomesenteric distance is normally between 10 and $28 \mathrm{~mm}^{2}$ A diagnosis of SMA syndrome may be made with a reduction of 6 to 160 in the angle and 2 to $8 \mathrm{~mm}$ in the aortomesenteric distance. ${ }^{3}$ SMA syndrome is a rare disease, with a prevalence ranging $0.013 \%$ to $0.3 \%$, although the syndrome is more common in females and patients between 10 and 39 years of age. ${ }^{1}$

Predisposing factors include significant weight loss, dietary disorders, scoliosis, post-operative conditions, trauma, congenital anomalies, and other local pathologies. ${ }^{2}$ The case above describes a typical presentation of SMA syndrome, involving nausea, vomiting, epigastric pain, loss of appetite, and weight loss. The 
differential diagnosis for SMA syndrome includes intraluminal intestinal obstruction, gastroesophageal reflux disease, irritable bowel syndrome, chronic gastritis, diverticulitis, small bowel obstruction, pancreatitis, colonic ileus, and duodenal obstruction from mesenteric stents. ${ }^{4}$

The initial treatment for SMA syndrome is generally nonsurgical and aims to build the aortomesenteric fat pad to increase space; this form of management has a success rate between $14 \%$ and $83 \% .^{5}$ As was implemented above, this treatment is administered via dietary changes in the form of small high-caloric meals and assumption of the left lateral decubitus position after meals.

Surgical options for treatment of SMA syndrome include gastrojejunostomy, duodenojejunostomy, Strong's procedure, and infrarenal transposition.

Gastrojejunostomy, or more specifically, a Roux-en-Y anastomosis, connects the stomach to the jejunum directly in order to decompress the stomach. However, as the duodenum remains connected and obstructed, this may result in blind loop syndrome and bile reflux. ${ }^{6}$ Further study needs to be done on success rates of gastrojejunostomies in treating SMA syndrome.

Duodenojejunostomy detaches the duodenum from the duodenojejunal junction and reattaches it to the jejunum distally, can be performed laparoscopically, and boasts a success rate of between $80 \%$ to $90 \%{ }^{7}$ However, complications of duodenojejunostomies include higher risk of bleeding, anastomotic leakage and stricture, and alterations to bilious circulation. ${ }^{8}$

Strong's procedure, or duodenal derotation, mobilizes the third and fourth portions of the duodenum and the duodenojejunal junction by dividing the ligament of Treitz, and then passes the jejunum through the superior mesenteric axis to the right of the vessels. This procedure does not modify the gastrointestinal tract, and success rates of up to $95 \%$ have been reported. ${ }^{8}$

Finally, infrarenal transposition of the superior mesenteric artery, where the superior mesenteric artery is moved inferiorly into the infrarenal aorta, has been performed to treat SMA syndrome as well. ${ }^{9}$ However, this procedure is not possible in a minimally invasive manner.

\section{Robot-Assisted Surgery}

Robot-assisted surgery (RAS) has been rising in popularity in several surgical specialties over the past decade due to the shift towards minimally invasive procedures. Although most surgeons currently have limited experience with RAS, there has been a great deal of research on patient outcomes after RAS.

Several reviews and meta-analyses have found RAS to be more effective than conventional surgery, although measures of success vary between procedures. A 2015 meta-analysis of robot-assisted versus open thoracic surgery for lung cancer found that RAS resulted in significantly lower perioperative morbidity and mortality. ${ }^{10} \mathrm{~A}$ review of pancreatic surgery in 2012 found comparable morbidity and mortality rates to those of open and conventional laparoscopic surgeries but also potentially higher rates of spleen preservation. ${ }^{11}$ A review of endometrial cancer surgery in 2016 found that RAS was associated with significantly lower blood loss, lower conversion rate, and a shorter hospital stay than conventional laparoscopic surgery. ${ }^{12}$

The outcomes of RAS in specific populations have been found to be equivalent to conventional surgery as well. A review of oncologic and general surgery in elderly patients in 2017 found no increased risk of death or morbidity compared to younger patients, although patients between 65 and 79 years of age had a higher rate of conversion to open surgery. ${ }^{13}$ Also, a 2017 review of pediatric abdominal and urologic surgery found that although there were no clear advantages to RAS, there were no statistical differences in complication rate either. ${ }^{14}$

However, studies have also reported findings to the contrary. A 2016 systematic review and meta-analysis of randomized control trials comparing RAS to conventional laparoscopic surgery found that, of 20 studies comprising 981 patients, there were no significant differences in blood loss, complication rates, or hospital stay, but operative times were significantly longer. The study concluded that RAS was not worth the significantly higher cost at the time. ${ }^{15}$

Furthermore, there are still problems with RAS systems. A 2008 review of the United States Food and Drug Administration (US FDA) Manufacturer and User Facility Device Experience Database (MAUDE) found a total of 189 device failures between January 1, 2000 and August 27, 2007, of which 4.8\% (9 cases) were associated with patient injury causing harm. However, the number of conversions to open surgery decreased with experience: of the adverse events reported, $94 \%$ were converted in 2003, as compared to $16 \%$ in $2007 .{ }^{16}$ A systematic review in 2013 also found that RAS could also result in port site metastases when used in carcinoma removal, although this only occurred in $2 \%$ of cases (5 of 204). ${ }^{17}$

In 2012, the Canadian Agency for Drugs and Technologies in Health conducted studies regarding the clinical and costeffectiveness of robot-assisted prostatectomies, hysterectomies, partial nephrectomies, and cardiac surgery in Canada. They found that although RAS was at least as clinically effective as conventional open or laparoscopic surgery in all four procedures, it was only slightly cost-effective in hysterectomies, and cost-neutral in cardiac surgery. ${ }^{18,19}$ In summary, more trials with longer follow-up times are necessary to better determine the clinical and cost-effectiveness of robot-assisted surgery. In addition, more development needs to be focused on reducing its cost and improving systems to better improve patient outcomes.

\section{CONCLUSION}

We present a case of superior mesenteric artery syndrome treated to full recovery by a robot-assisted Strong's procedure. Our case and discussion call to attention a rare syndrome and the multiple treatment avenues available for it, all of which require careful patient-specific consideration. Our case thus highlights both the difficulty and the importance of diagnosing and treating SMA syndrome.

Furthermore, given the current debate on the effectiveness of robot-assisted surgery, our case presents a timely discussion on the benefits and drawbacks of RAS. We conclude that in its current state, RAS follows the paradigm shift towards minimally invasive procedures but has varying degrees of success and may not be costeffective for widespread use in Canada.

Page 76 


\section{REFERENCES}

1. Konstantinidis H, Charisis C, Kottos P. Robotic Strong's procedure for the treatment of superior mesenteric artery syndrome. Description of surgical technique on occasion of the first reported case in the literature. Int J Med Robot. 2018 Feb; 14(1): e1876. https://doi.org/10.1002/rcs.1876

2. Derric JR, Fadhli HA. Surgical anatomy of the superior mesenteric artery. Am Surg. 1965 Aug;31:545-7.

3. Welsch T, Bühler MW, Kienle P. Recalling superior mesenteric artery syndrome. Dig Surg. 2007;24(3):149-56. https://doi.org/10.1159/000102097

4. Dynamed Plus. Superior Mesenteric Artery Syndrome [Internet]. Ipswich (MA): EBSCO Information Services; May 2017 [cited 2018 Mar 15]. Available from: http://www.dynamed.com/topics/ dmp AN T114160/Superior-mesenteric-artery-syndrome.

5. Shin MS, Kim JY. Optimal duration of medical treatment in superior mesenteric artery syndrome in children. J Korean Med Sci. 2013 Aug;28(8):1220-5. https://doi.org/10.3346/jkms.2013.28.8.1220

6. Lee CS, Mangla JC. Superior mesenteric artery compression syndrome. Am J Gastroenterol. 1978 Aug;70(2):141-50.

7. Raissi B, Taylor B, Traves D. Recurrent mesenteric artery (Wilkie's) syndrome: a case report. Can J Surg. 1996 Oct;39(5):410-6.

8. Ha CD, Alvear DT, Leber DC. Duodenal derotation as an effective treatment of superior mesenteric artery syndrome: a thirty-three year experience. Am Surg. 2008 Jul;74(7):644-53.

9. Pourhassan S, Grotemeyer D, Fürst G, et al. Infrarenal transposition of the superior mesenteric artery: a new approach in the surgical therapy for Wilkie syndrome. J Vasc Surg. 2008 Jan;47(1):201-4. https://doi.org/10.1016/j.jvs.2007.07.037

10. Zhang L, Gao S. Robot-assisted thoracic surgery versus open thoracic surgery for lung cancer: a system review and meta-analysis. Int J Clin Exp Med. 2015 Oct;8(10):17804-10. https://doi.org/10.1093/icvts/ivs472

11. Strijker M, van Santvoort HC, Besselink MG, et al. Robot-assisted pancreatic surgery: a systematic review of the literature. HPB (Oxford). 2013 Jan;15(1):1-10 https://doi.org/10.1111/j.1477-2574.2012.00589.x

12. Xie W, Cao D, Yang J, et al. Robot-assisted surgery versus conventional laparoscopic surgery for endometrial cancer: a systematic review and meta-analysis. J Canc Res Clin Oncol. 2016 Oct;142(10):2173-83. https://doi.org/10.1016/j.tjog.2016.01.003

13. Ceccarelli G, Andolfi E, Biancafarina A, et al. Robot-assisted surgery in elderly and very elderly population: our experience in oncologic and general surgery with literature review. Aging Clin Exp Res. 2017 Feb;29(Suppl 1):55-63. https://doi.org/10.1007/s40520-016-0676-5

14. Lima M, Michela M. Robot-assisted pediatric surgery: review of comparative studies focusing on complications. JEMIS. 2017 Jun;5(1):1-9. https://doi.org/10.1473/JEMIS28

15. Broholm M, Onsberg Hansen I, Rosenberg J. Limited evidence for robot-assisted surgery: a systematic review and meta-analysis of randomized controlled trials. Surg Laparosc Endosc Percutan Tech. 2016 Apr;26(2):117-23. https://doi.org/10.1097/SLE.0000000000000248

16. Andonian S, Okeke Z, Okeke DA, et al. Device failures and patient injuries associated with robot-assisted laparoscopic surgeries: a review of the FDA database. Can J Urol. 2008 Feb;15(1):3912-6.

17. Iavazzo C, Gkegkes ID. Port site metastases after robot-assisted surgery: a systematic review. Int J Med Robot Comput Assist Surg. 2013 Jun;9:423-7. https://doi.org/10.1002/rcs.1512
18. Canadian Agency for Drugs and Technology in Health. Robotassisted surgery for prostatectomy and hysterectomy: a review of the clinical and cost-effectiveness - an update. [Internet]. CADTH Rapid Response Service; 2012 [cited 2018 Mar 15]. Available from: https://www.cadth.ca/media/pdf/htis/nov-2012/RC0400\%20Robotassisted\%20prostatectomy\%20and\%20hysterectomy\%20Final.pdf.

19. Canadian Agency for Drugs and Technology in Health. Robot-assisted surgery for partial nephrectomy and cardiac surgery: a review of the clinical and cost-effectiveness - an update. [Internet]. CADTH Rapid Response Service; 2012 [cited 2018 Mar 15]. Available from: https:// www.cadth.ca/media/pdf/htis/nov-2012/RC0401\%20-\%20RobotAssisted\%20Nephrectomy\%20and\%20Cardiac\%20Surgery\%20Final. pdf. 